\title{
What does acute dyspnea hide?
}

\author{
Giovanna Squiccimarro - Maria Carrabba • \\ Cinzia Hu • Maria Domenica Cappellini • \\ Giovanna Fabio
}

Received: 18 May 2011/ Accepted: 21 June 2011/Published online: 13 July 2011

(C) SIMI 2011

\section{Case presentation}

Dr. Giovanna Squiccimarro In December 2009, a previously healthy 48-year-old man was admitted because of hypertensive crisis and bilateral diffuse ground-glass shadows on the chest X-ray study.

He presented at the Emergency Department (ED) complaining of worsening dyspnea.

The respiratory rate was 35 breaths/min and $\mathrm{SpO} 293 \%$ at pulse oximeter. His blood pressure (BP) was 220/120 $\mathrm{mmHg}$ and the heart rate 120 beats/min.

He was overweight, afebrile and diaphoretic. He had a history of alcohol and tobacco abuse (more than 20 cigarettes/day). He denied use of any medications, and reported that mild dyspnea and fever (TA $39^{\circ} \mathrm{C}$ ) acutely began 3 weeks prior. The fever resolved without any therapy in a few days but a mild dyspnea persisted.

Ten days prior to admission, dyspnea was still present and an isolated hemoptysis occurred.

Normal lung breath sounds and a 3/6 heart systolic murmur were heard on the examination. The electrocardiogram (EKG) showed sinus tachycardia, right bundlebranch block and S1-Q3 pattern.

G. Squiccimarro and M. Carrabba contributed equally to this work.

M. Carrabba - C. Hu - M. D. Cappellini - G. Fabio

Department of Internal Medicine, UO Medicina Interna 1-A, Università degli Studi di Milano, Fondazione IRCCS

"Cà Granda" Ospedale Maggiore Policlinico,

Via F. Sforza 35, 20122 Milan, Italy

\section{G. Squiccimarro $(\square)$}

Department of Internal Medicine, UO Medicina Interna 1-A, Università degli Studi di Milano, Scuola di Specializzazione in Medicina Interna, Via F. Sforza 35, 20122 Milan, Italy e-mail: giosquicci@libero.it
Arterial blood gas (ABG) analysis while breathing room air showed pH 7.47, $\mathrm{PaO} 257 \mathrm{mmHg}, \mathrm{pCO} 233 \mathrm{mmHg}$, $\mathrm{HCO} 3$ - $25.4 \mathrm{mmol} / \mathrm{L}$. Laboratory tests were normal except for WBC count $10,200 / \mathrm{mmc}(N=80 \%)$, C-RP $1.31 \mathrm{mg} / \mathrm{dL}$ (normal range $<0.5$ ), creatinine $1.4 \mathrm{mg} / \mathrm{dL}$, $\mathrm{K}^{+} 3.0 \mathrm{mEq} / \mathrm{L}, \quad \mathrm{LDH} 493 \mathrm{U} / \mathrm{L}$ (normal range $<480$ ), D-dimer $438 \mu \mathrm{g} / \mathrm{mL}$ (normal range $<250$ ).

Because of his symptoms and signs, patient was scored for suspected acute pulmonary embolism (PE). An intermediate clinical risk for PE was assessed by both Wells' and revised Geneva scores. Considering the positivity of the D-dimer test, he was imaged with the chest CT-scan, according to the diagnostic work-up for the management of suspected pulmonary embolism.

The CT-scan revealed bilateral diffuse ground-glass opacities mostly in the right lung, and multifocal bilateral consolidations resembling diffuse inflammatory processes (Fig. 1). Mediastinal and paratracheal lymphadenopathies were found. There were bilateral pleural effusions. No pulmonary embolism was found.

Empirical wide spectrum antibiotic therapy and oxygen therapy were given and intravenous nitroglycerin was started promptly. Then patient was hospitalized for further investigations.

\section{Differential diagnosis}

Dr. Maria Carrabba (Internist) A pulmonary embolism was suspected because of the dyspnoea, ABG analysis, EKG findings and D-dimer elevation. A CT-scan revealed bilateral diffuse ground-glass opacities and multifocal bilateral consolidations resembling diffuse inflammatory processes. $\mathrm{Dr}$. Hu, what is your opinion? Which diagnoses are consistent with these findings? Also why was the D-dimer elevated? 
Fig. 1 CT-scan exhibits bilateral disease with groundglass opacities and airspace consolidation of lung parenchyma (a, b). Bilateral pleural effusion $(\mathbf{c}, \mathbf{d})$ and paratracheal lymphadenopathies are also evident (b)
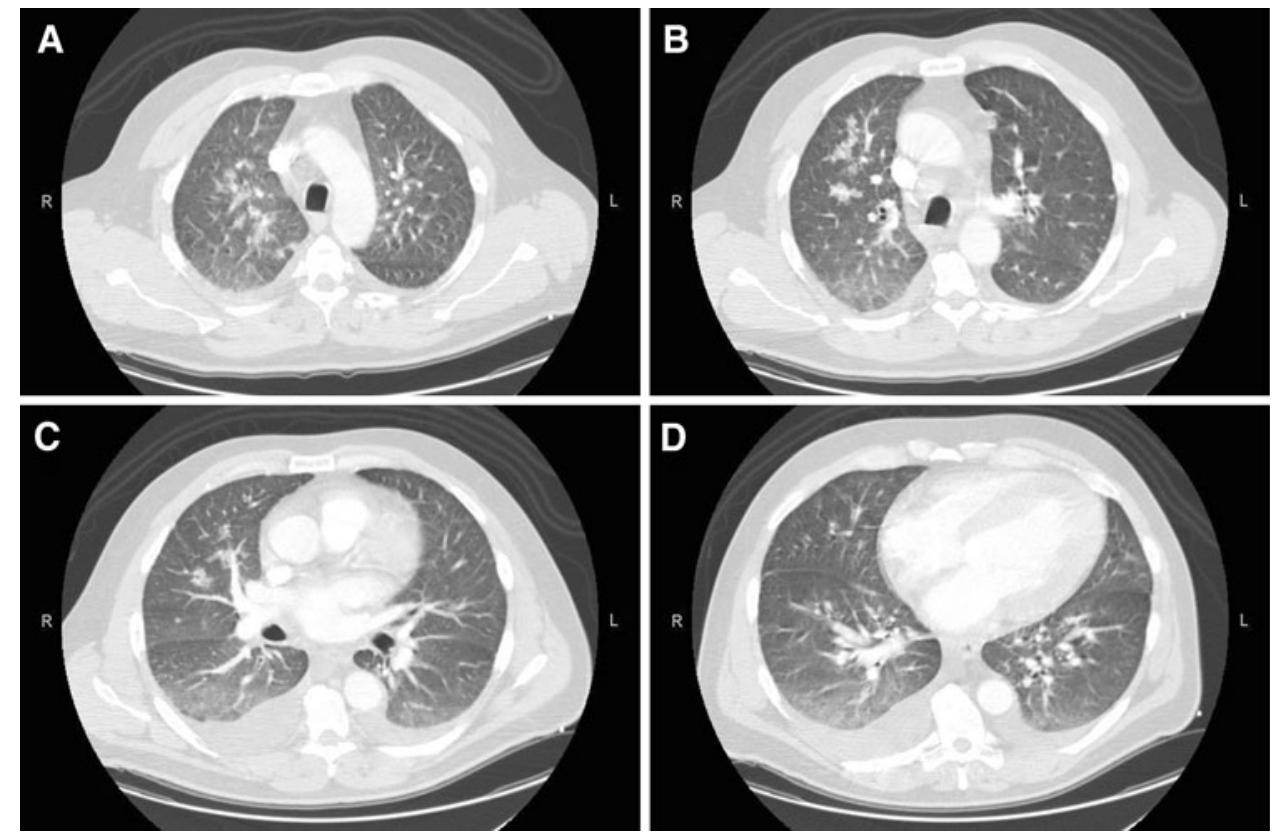

Dr. Cinzia Hu (Internist and Infective disease specialist) In this patient, the clinical probability of PE was intermediate given the tachypnoea, pleuritic chest pain, recent hemoptysis, ABG analysis and EKG findings. Performing a chest CT-scan was mandatory [1]. The CT-scan gave us another key for evaluating this patient's clinical picture: multifocal bilateral inflammatory processes.

I believe that the increased D-dimer value can be attributed to an inflammatory response rather than to a thromboembolic event. Also the ABG analysis at the admission was probably related to the ground-glass pattern and the patient's high respiratory rate.

Reviewing this patient's history, he presented with a flu-like syndrome 3 weeks before admission. Then he experienced a single case of hemoptysis and increasing dyspnoea.

I think that in this patient, an interstitial pneumonia can be hypothesized on the basis of several clinical signs and the CT-scan findings. An interstitial pattern is associated with the so-called atypical pneumonias, which can be caused by organisms such as Mycoplasma or Chlamydia. They often present with an influenza-like illness and dry cough. Radiological investigations show diffuse interstitial infiltrates, usually not consolidation. Legionella pneumonia is found in $2-25 \%$ of adults hospitalized for pneumonia. At radiological investigation, there are commonly focal infiltrates that are poorly demarcated. However, some studies have demonstrated an alveolar pattern of shadowing in the initial stages in up to $81 \%$ of patients, not the classically taught interstitial shadowing [2].

Additional infectious agents include viruses, (e.g., influenza virus, adenovirus, respiratory syncytial virus and varicella zoster virus), mycobacteria, pneumocystis, parasites and fungi (e.g., coccidioidomycosis, Aspergillus spp.). Primary viral pneumonia typically occurs after the onset of classical influenza symptoms with rapid deterioration. The chest radiograph can show a ground-glass opacity or linear shadowing. As the disease progresses, patchy consolidations can appear on the radiograph that become confluent over time with nodular opacities also seen. More than half of adults' viral pneumonias are found to be caused by influenza virus types A and B [3]. Since it was flu epidemic time, the ground-glass shadow pattern could be ascribed to A H1N1 virus.

The multifocal bilateral consolidations and the slightly increased serum inflammatory markers might also suggest a fungal infection, in a patient with an underlying immunocompromise. Hence the competence of the immune system should also be evaluated.

Differentiation of infectious pneumonia from noninfectious syndromes may be difficult at presentation, thus in the presence of opacities on a chest radiograph, it is prudent to start broad-spectrum antibiotics. The only definitive way to reach a specific diagnosis is through demonstration of the infecting organism. It should be obtained through a microscopic or molecular examination of stained smears of sputum or pleural fluid or other biological material, or a culture of respiratory secretions or blood.

Hence, when I saw the patient, even though he was on wide-spectrum antibiotics, I decided to perform wide pneumonia screening and comprehensive molecular tests for H1N1 and adenovirus. HIV was added to exclude another cause of immunocompromise. Unfortunately, no cultures or staining by biological fluids were obtained. 
Because of the pericardial effusion and the EKG alteration, serological tests for pericarditis and myocarditis were also performed.

Dr. Maria Carrabba The molecular tests for H1N1 and HIV Ag/Ab detection were negative. Urinary Ag-detection and blood tests for common atypical pneumonia pathogens were negative. Serological tests for Aspergillus spp., CMV, EBV, ECHO virus, Coxsackie, Adenovirus, Parvovirus were negative for acute infection. An infectious etiology of the interstitial lung disease was excluded, but there were many other clinical problems to consider.

Dr. Giovanna Squiccimarro In addition to the lung abnormalities, in the first day after admission, we had to deal with his severe hypertension. Indeed, after $48 \mathrm{~h}$ of nitroglycerin endovenous therapy, his BP was still 180/100 $\mathrm{mmHg}$ and EKG alterations persisted. He also had a slight elevation in creatinine levels and a mild proteinuria. An echocardiogram showed left ventricle severe hypertrophy, mild pulmonary hypertension, severe mitral and moderate tricuspid regurgitations and a small pericardial effusion. Hence, a chronic hypertensive status, not reported by the patient, was suspected. Moreover, considering his severe BP elevation, a secondary hypertension was hypothesized. Renovascular hypertension is the most common correctable cause of secondary hypertension. It accounts for less than $1 \%$ of cases of mild-to-moderate elevations in BP. However, the incidence of this disorder markedly rises in patients with acute (even if superimposed upon a preexisting elevation in BP), severe, or refractory hypertension [4].

Primary aldosteronism, hypothyroidism, Cushing's syndrome, pheochromocytoma, sleep apnoea, and coarctation of the aorta were also considered. On the physical examination, we found no clues suspected for Cushing's syndrome. Echocardiogram and CT-lung scan excluded aorta coarctation. A renal artery Doppler ultra sound study (US) ruled out renovascular hypertension. Then laboratory evaluation was necessary for ruling out hypothyroidism, hyperaldosteronism or glucocorticoids excess. Thyroid hormonal assessment was normal as were the plasma catecholamines and renin. Aldosterone dosage was slightly increased $(167 \mathrm{pg} / \mathrm{ml}$; normal range $<150 \mathrm{pg} / \mathrm{ml})$ with a normal plasma potassium concentration. The low ratio of the plasma aldosterone concentration to plasma renin activity ruled out a diagnosis of primary aldosteronism [5]. We planned further evaluation for sleep apnoea.

Dr. Maria Carrabba Thus, the patient seemed to have a noninfectious interstitial pneumonia with mediastinal lymph nodes, and a severe hypertension of undefined origin. Dr. Fabio, how do you think all these findings could be brought together?

Dr. Giovanna Fabio (Internist and Immunologist) The bilateral ground-glass opacities seen on the chest CT-scan represent alveolar filling, which points to a differential diagnosis that includes infection, edema, interstitial lung disease, diffuse alveolar hemorrhage and cancer.

Interstitial lung diseases can complicate the course of most of the connective tissue diseases (e.g., polymyositis/ dermatomyositis, rheumatoid arthritis, systemic lupus erythematosus, scleroderma, Sjögren's syndrome, mixed connective tissue disease) [6], and include conditions such as ANCA positive systemic vasculitides, sarcoidosis, alveolar proteinosis, chronic eosinophilic pneumonia, idiopathic pulmonary fibrosis and other less common forms of "idiopathic" interstitial lung diseases, occupational or environmental exposures (e.g., smoking, dusts, and hypersensitivity pneumonitis), drug-induced and radiationinduced lung injury [7].

In this patient, vasculitis could be a good explanation both for interstitial pneumonitis and severe hypertension. Low values of both ERS (19 mm/h) and C-RP and normal urinary sediment findings made unlikely the diagnoses of vasculitis or connective tissue diseases, nevertheless antinuclear and ANCAs antibodies should be performed.

The absence of asthma, purpura and eosinophilia rules out Churg-Strauss and hypereosinophilic syndrome. Also allergic bronchopulmonary aspergillosis, (caused by an IgE-mediated hypersensitivity reaction to aspergillus) is unlikely in this case, in the absence of asthma.

Sarcoidosis can present with hilar lymphadenopathy and pulmonary infiltrates, similar to the patient's radiological findings. The diagnosis of sarcoidosis is established on the basis of compatible clinical and radiologic findings, supported by histologic evidence in one or more organs of noncaseating epithelioid-cell granulomas in the absence of organisms or particles [8].

The most important distinction among the idiopathic interstitial pneumonias is between idiopathic pulmonary fibrosis and the other interstitial pneumonias. The latter includes: nonspecific interstitial pneumonia, desquamative interstitial pneumonia, respiratory bronchiolitis-associated interstitial lung disease, acute interstitial pneumonia, cryptogenic organizing pneumonia, and lymphocytic interstitial pneumonia [9]. Nonspecific interstitial pneumonia is identical to idiopathic pulmonary fibrosis in its clinical features at presentation, although its association with smoking is not well established. Patients with nonspecific interstitial pneumonia tend to have less fibrosis and more ground-glass opacities than those with usual interstitial pneumonia, but there is considerable overlap.

In this patient, we have to consider also bronchiolitis obliterans with organizing pneumonia (BOOP) that may be idiopathic or may be due to an infection, transplantation, or collagen vascular disease. An influenza-like prodrome is seen in $24 \%$ of the patients, and the disease may be rapidly progressive in previously healthy persons. The patient's age, the presentation with cough, dyspnea, and previous 
fever made likely both diagnosis of BOOP and idiopathic interstitial pneumonia.

The patient had mediastinal lymph nodes borderline in size. The CT-scan findings were suggestive but not diagnostic of a malignant tumor. Bronchoalveolar-cell carcinoma and non-Hodgkin's lymphoma may mimic pneumonia. However, the patient had no findings on the physical examination such as splenomegaly or lymphadenopathy.

Neoplastic diseases may present with multifocal involvement, although nodules and masses are more typical. The onset is subacute, as in this case, sometimes associated with fever and weight loss. Signs such as hypertension, as well as hypercalcemia or others, could be the expression of an associated endocrine paraneoplastic syndrome. Carcinomas of the lung are one of the most common tumors causing ectopic hormone secretion [10]. I think that malignancy, and especially lung cancer, should also be considered among the possible causes of this patient's illness.

A bronchoscopy with bronchoalveolar lavage (BAL), and eventually endobronchial or transbronchial lung biopsy (TBLB) could be the next step in diagnosis [11]. In the correct clinical setting, typical BAL findings are diagnostic in several rare diffuse lung diseases, including alveolar proteinosis, lipoid pneumonia, acute eosinophilic pneumonia, pulmonary lymphoma and lymphangitis carcinomatosis (although in the last disorder, the diagnosis is usually also apparent at TBLB). In fibrosing disorders, a BAL neutrophilia, often associated with an eosinophilia, is common. Granulomatous disease, by contrast, is characterized by the predominance of a BAL lymphocytosis, with a variable BAL neutrophil content and an occasional BAL eosinophilia is common in hypersensitivity pneumonitis.

TBLB can be useful in diagnosing neoplasia, infections and certain interstitial lung diseases. In interstitial lung diseases, TBLB is recommended in those with prominent bronchocentric involvement, such as lymphangitis carcinomatosis or sarcoidosis. In sarcoidosis, TBLB has a diagnostic yield of at least $85 \%$ when multiple lung segments are sampled. TBLB is not recommended in suspected idiopathic pulmonary fibrosis, except to demonstrate an alternative diagnosis, such as sarcoidosis or hypersensitivity pneumonitis. TBLB is equally unhelpful in the diagnosis of idiopathic interstitial pneumonia, except to confirm cryptogenic organizing pneumonia or hypersensitivity pneumonitis, in which TBLB are not diagnostic in isolation, but in combination with the clinical and CT findings.

However, careful documentation of the past medical history is important in the initial assessment of interstitial lung diseases, as the cause of the illness is often recognized from the patient's medical history. Attention is also given to history of smoking, medication use, occupational and environmental exposures, and familial occurrence.

I think that patients' habits could be the key of this puzzle. I believe that, before we plan invasive diagnostic investigations such as bronchoscopy with BAL and eventually TBLB, it is of great importance to question the patient about occupation and what substances he might be exposed to. This patient was a smoker, but not quizzed for environmental or toxic exposures likely to cause hypersensitivity pneumonitis or lung injury. He should be asked also for illicit agents use.

\section{Further investigations}

Dr. Squiccimarro Additional history was obtained. The patient was a hydraulic worker and he declared himself to be a heavy smoker. He denied any use of drugs or exposure to toxic agents. He was asked also for travels, but in the prior years he did not travel abroad.

Finally, he was asked for illicit agents use, and he reported being a habitual daily cocaine user for 10 years. Because of cocaine abuse, along with the EKG and echocardiogram abnormalities, even if the patient denied any anginal event, a coronarography was performed. No coronary stenosis was found; the left ventricular dysfunction and severe mitral regurgitation were confirmed.

\section{Clinical diagnosis}

A diagnosis of Crack lung syndrome was made.

\section{Therapy and follow-up}

Dr. Carrabba The patient was started with empirical antibiotic therapy under the hypothesis of communityacquired interstitial pneumonia: 1-week course of oral azithromycin (500 mg od) and intravenous cephtriaxon (2 g od). He improved rapidly, and oxygen therapy was stopped after 2 days of hospitalization. At discharge he had completely recovered, and room air oxygen saturation was $97 \%$. Bronchoalveolar lavage was not performed because the chest CT-scan findings were consistent with the declared chronic cocaine abuse. Four drug therapies were started, after blood test for endocrine assessment. The BP levels normalized only after the commencement of diuretics, sartanics, Ca-antagonists and alpha-litics. The patient was discharged with good BP control. We lost him to follow up because he chose to be referred to another center for cardiological and pneumological monitoring. 


\section{Discussion}

Dr. Carrabba Pulmonary complications of cocaine are influenced by the method of administration, dose size, and the presence of associated substances. Heavy and repeated aspiration of cocaine and adulterants mixtures causes lung tissue damage resulting in many pulmonary and cardiovascular diseases.

Crack cocaine is the illicit substance most commonly associated with respiratory complications requiring hospital admission. Cough, hemoptysis, pneumothorax, pneumomediastinum, pneumopericardium, haemothorax, hilar lymphadenopathy, pulmonary edema, "crack lung”, interstitial pneumonitis, and BOOP are the main complications of inhaling crack cocaine vapor.

"Crack lung" is the name of a syndrome characterized by acute dyspnea (44\%), cough with sputum production (61\% of cases), interstitial pneumonitis or fibrosis $(38 \%)$, hemoptysis (6-26\%), diffuse pulmonary infiltrates, fever, and hypoxemia [12].

A wide spectrum of lung CT-scan imaging may be found in crack lung syndrome patients [13]. Pulmonary embolism can be misdiagnosed because of the cocaine vasoconstrictive effect on the pulmonary vasculature.

CT-scan opacities ground-glass could be signs of hemoptysis or occult pulmonary hemorrhage, secondary to rupture of alveolar, bronchial or tracheal submucosal blood vessels. Diffuse and occult alveolar hemorrhage in habitual cocaine smokers may be more common than clinically recognized [14]. The diagnosis is based upon a history of cocaine use and compatible clinical, radiological and, if needed, bronchoscopic findings.

Histologically [15], at autopsy, cocaine use is associated with alveolar hemorrhage, hemosiderin-laden macrophages from previous hemorrhage, congestion and edema, pneumonitis and interstitial fibrosis. Medial hypertrophy of the muscular small and medium-sized pulmonary arteries has been demonstrated. Carbon pigmented macrophages are common, and may be seen on bronchoalveolar lavage and in pleural effusions. Pulmonary edema is common in cocaine use, but appears to have a different composition to the pulmonary edema seen in opiate deaths. Because it is cardiogenic, rather than neurogenic in origin, it has low protein content and does not froth like opiate-induced pulmonary edema.

Dr. Fabio Indeed, cocaine potent sympathomimetic action activates endothelium, inducing damage to pulmonary microvasculature and platelets aggregation. Increased levels of alveolar macrophages hemosiderin as well as endothelin (ET)-1, a powerful vasoconstrictor, are found in cocaine-users bronchoalveolar fluid, and are considered to be markers of alveolar injury [16]. The cocaine-mediated increase of ET-1 release, and the decreased production of nitric oxide, which is a vasodilator, affect endothelial leukocyte interactions, smooth cells contraction and induce platelet activation and hyperaggregability. All the above events lead to leakage of fluid and erythrocytes from injured pulmonary capillaries into the alveoli [16].

Therefore, the lungs and endothelium become the principal organs exposed and affected, and the majority of cocaine abusers manifest pulmonary hypertension, high blood pressure and atherosclerosis.

By increasing heart rate, blood pressure, and contractility, cocaine leads to increased myocardial demand. Myocardial ischemia and infarction may be ongoing even in the absence of chest pain, being masked by the adulterants (lidocaine, benzocaine) commonly used to cut cocaine. Although still controversial, beta-blockers should be considered for patients with cocaine-related coronary artery disease or left ventricular dysfunction [17].

Crack users may develop adult respiratory distress syndrome and end-stage respiratory failure due to crackassociated interstitial pneumonitis and BOOP. Cessation of crack cocaine use is mandatory. Systemic corticosteroid therapy for interstitial pneumonitis is still debated, even if the lung injury may respond when a prominent inflammatory cell infiltration is present $[12,14]$.

Follow-up of these patients demonstrates that, despite discontinuation of drug abuse, interstitial fibrosis may progress with the development of respiratory insufficiency and significant mortality [14].

\section{Conclusion}

The possibility of cocaine use should be considered in any adult admitted to the ED with acute dyspnea or hemoptysis, who has an interstitial pneumonia even in the absence of symptoms and signs of acute myocardial ischemia. Establishing a diagnosis of acute crack lung syndrome in the emergency setting may influence diagnostic and therapeutic strategies for immediate therapy and may help to prevent chronic damages.

Acknowledgments This case was selected from the "Casi Clinici Gymnasium" abstracts submitted to the 2010 SIMI Annual Congress in Rome, and was reviewed by a Committee composed of Nicola Montano and Luigi Pagliaro.

Conflict of interest None.

\section{References}

1. Agnelli G, Becattini C (2010) Current concepts: acute pulmonary embolism. N Engl J Med 363:266-274

2. Godet C, Frat J, Le Moal G, Roblot F, Michalakis G, Cabon E, Tasu JP (2007) Legionnaire's pneumonia: is there really an interstitial lung disease? Eur J Radiol 61:150-153 
3. Shiley KT, Van Deerlin VM, Miller WT Jr (2010) Chest CT features of community-acquired respiratory viral infections in adult inpatients with lower respiratory tract infections. J Thorac Imaging 25(1):68-75

4. Mann SJ, Pickering TG (1992) Detection of renovascular hypertension. State of the art: 1992. Ann Intern Med 117:845

5. Ahmed AH, Gordon RD, Taylor P, Ward G, Pimenta E, Stowasser M (2010) Effect of atenolol on aldosterone/renin ratio calculated by both plasma Renin activity and direct Renin concentration in healthy male volunteers. J Clin Endocrinol Metab 95:3201

6. Castelino FV, Varga J (2010) Interstitial lung disease in connective tissue diseases: evolving concepts of pathogenesis and management. Arthritis Res Ther 12:213

7. Dempsey OJ, Kerr KM, Remmen H, Denison AR (2010) How to investigate a patient with suspected interstitial lung disease. BMJ 340:c2843. doi:10.1136/bmj.c2843

8. Iannuzzi MC, Rybicki BA, Teirstein AS (2007) Sarcoidosis. N Engl J Med 357:2153-2165

9. American Thoracic Society, European Respiratory Society (2002) American Thoracic Society/European Respiratory Society international multidisciplinary consensus classification of the idiopathic interstitial pneumonias. Am J Respir Crit Care Med 165:277-304

10. DeLellis RA, Xia L (2003) Paraneoplastic endocrine syndromes: a review. Endocr Pathol 14(4):303-317
11. Reynolds HY (2009) Present status of bronchoalveolar lavage in interstitial lung disease. Curr Opin Pulm Med 15(5):479-485

12. Devlin RJ, Henry JA (2008) Clinical review: major consequences of illicit drug consumption. Crit Care 12:202

13. Restrepo CS, Carrillo JA, Martìnez S et al (2007) Pulmonary complications from cocaine and cocaine-based substances: imaging manifestations. Radiographics 27:941-956

14. Baldwin GC, Choi R, Roth MD, Shay AH, Kleerup EC, Simmons MS, Tashkin DP (2002) Airway effects of marijuana, cocaine, and other inhaled illicit agents pulmonary microcirculation in habitual users of alkaloidal ("crack") cocaine. Chest 121:1231-1238

15. Milroy CM, Parai JL (2011) The histopathology of drugs of abuse. Histopathology. doi:10.1111/j.1365-2559.2010.03728.x. [Epub ahead of print]

16. Pradhan L, Mondal D, Chandra S, Ali M, Agrawal KC (2008) Molecular analysis of cocaine-induced endothelial dysfunction: role of endothelin-1 and nitric oxide. Cardiovasc Toxicol 8:161-171

17. McCord J, Jneid H, Hollander JE et al (2008) Management of cocaine-associated chest pain and myocardial infarction a scientific statement from the American heart association acute cardiac care committee of the council on clinical cardiology. Circulation 117:1897-1907 